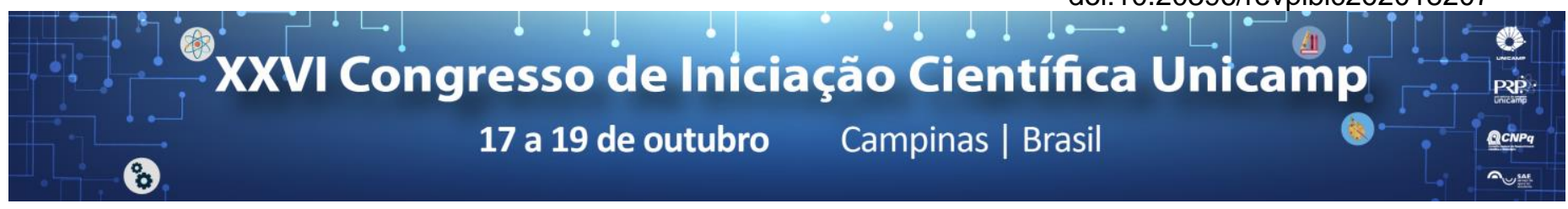

\title{
Um Estudo sobre Métodos de Apoio à Decisão em Grupo
}

\section{Beatriz V. B. S. de Souza*, Leonardo Tomazeli Duarte}

\section{Resumo}

Em um processo de decisão em grupo, indivíduos estão diante de questões específicas em que todos estão interessados em obter uma solução que atenda suas expectativas. Sob à perspectiva dos métodos de apoio à decisão existentes, esse processo se torna complexo e desafiador, devido a possível existência de conflitos de interesses e à necessidade de considerar as preferências individuais na busca por uma decisão coletiva. O presente projeto visa apresentar alguns dos resultados obtidos em um estudo acerca de alguns métodos de apoio à decisão em grupo, a saber: AHP, PROMETHEE e um método para cálculo de consenso baseado em distâncias.

\section{Palavras-chave:}

Decisão em Grupo, MCDA, Consenso.

\section{Introdução}

No contexto organizacional, a exigência por decisões assertivas em um curto espaço de tempo é enorme e, nesse sentido, a tomada de decisão em grupo assume um papel importante, sendo essencial 0 uso de ferramentas de suporte à decisão [3]. Existe um conjunto de métodos de apoio à decisão multicritério (MCDA) que proporcionam uma representação multidimensional dos problemas, através da avaliação de um conjunto finito de alternativas sob múltiplos critérios [3]. Entre eles, estão o método AHP e PROMETHEE. Em um modelo de decisão com base no consenso, os decisores expressam suas opiniões, mas abrem mão de suas preferências individuais em prol de uma solução que seja razoável para todos do grupo [1]. Um dos métodos dessa abordagem é o método baseado em distâncias [2].

\section{Resultados e Discussão}

Para compreender a aplicabilidade dos três métodos de apoio à decisão em grupo estudados, eles foram comparados através de um programa criado no software Matlab. Os dados da avaliação das alternativas sob os critérios foram gerados a partir de uma simulação de Monte Carlo, através de uma função de distribuição normal multivariada. Esta função é descrita por meio de uma matriz de covariância e pela média entre os elementos (Figura 1). A análise foi feita através de 3 cenários, no primeiro, um decisor outlier, no segundo, sem relação entre eles e, no terceiro, três decisores são relacionados.

$$
\begin{array}{cc}
\operatorname{Cov} 1=\left[\begin{array}{ccc}
1 & -0,5 & 0,3 \\
-0,5 & 1 & 0 \\
0,3 & 0 & 1
\end{array}\right] & \operatorname{Cov} 2=\left[\begin{array}{lll}
1 & 0 & 0 \\
0 & 1 & 0 \\
0 & 0 & 1
\end{array}\right] \quad \operatorname{Cov} 3=\left[\begin{array}{ccc}
1 & 0,8 & 0,8 \\
0,8 & 1 & 0,8 \\
0,8 & 0,8 & 1
\end{array}\right] \\
\text { Média } 1=\left[\begin{array}{lll}
3 & 3 & 3
\end{array}\right] & \text { Média } 2=\left[\begin{array}{lll}
3 & 3 & 3
\end{array}\right] \quad \text { Média } 3=\left[\begin{array}{lll}
3 & 3 & 3
\end{array}\right]
\end{array}
$$

Figura 1. Covariância e média dos 3 cenários.

Utilizou-se um modelo de decisão com 500 alternativas, 3 critérios e 3 decisores. A comparação foi feita par a par entre os métodos, de acordo com suas medidas de correlação e distância de Kendall tau. Os resultados médios dessas comparações seguem na Tabela 1.

Tabela 1. Resultados das comparações.

\begin{tabular}{|l|c|c|c|c|c|c|}
\hline \multirow{2}{*}{ Comparações } & \multicolumn{3}{c|}{ Correlação Média } & \multicolumn{3}{c|}{ Kendall Tau médio } \\
\cline { 2 - 8 } & Cenário 1 & Cenário 2 & Cenário 3 & Cenário 1 & Cenário 2 & Cenário 3 \\
\hline AHP e PROMETHEE & 0,9395 & 0,9475 & 0,9681 & 0,0942 & 0,0914 & 0,0480 \\
\hline AHP e Consenso & 0,9885 & 0,9923 & 0,9996 & 0,0395 & 0,0342 & 0,0084 \\
\hline PROMETHEE e Consenso & 0,9518 & 0,9561 & 0,9676 & 0,0874 & 0,0863 & 0,0493 \\
\hline
\end{tabular}

O coeficiente de correlação de Pearson estabelece uma medida de relação entre os dados. A distância de Kendall tau é uma métrica que contabiliza o número de discordâncias entre os ordenamentos obtidos por dois métodos diferentes. Analisando a Tabela 1, percebe-se que o par AHP e Consenso possuem o maior valor de correlação nos três cenários e a menor distância de Kendall Tau e, portanto, é o mais correlacionado. Já o par AHP e PROMETHEE possui os resultados mais descorrelacionados nos cenários 1 e 2 . No cenário 3, no entanto, é o par PROMETHEE e Consenso que possui os resultados mais distantes.

Assim, é possível inferir que os métodos AHP e Consenso, no geral, produzem resultados de decisão mais próximos e que o método de consenso se comporta melhor em cenários mais diversos do que em cenários com decisores mais parecidos.

Tabela 2. Desvio padrão entre as comparações.

\begin{tabular}{|l|c|c|c|c|c|c|}
\hline \multirow{2}{*}{\multicolumn{1}{|c|}{ Comparações }} & \multicolumn{2}{|c|}{ Desvio Padrão - Correlação } & \multicolumn{2}{c|}{ Desvio Padrão - Kendall Tau } \\
\cline { 2 - 7 } & Cenário 1 & Cenário 2 & Cenário 3 & Cenário 1 & Cenário 2 & Cenário 3 \\
\hline AHP e PROMETHEE & 0,0080 & 0,0055 & 0,0042 & 0,0059 & 0,0051 & 0,0031 \\
\hline AHP e Consenso & 0,0021 & 0,0014 & 0,0003 & 0,0030 & 0,0027 & 0,003 \\
\hline PROMETHEE e Consenso & 0,0050 & 0,0045 & 0,0045 & 0,0051 & 0,0053 & 0,0036 \\
\hline
\end{tabular}

\section{Conclusões}

Através das comparações dos métodos, foi possível verificar que, apesar das diferenças apresentadas, os métodos se mostraram robustos, uma vez que, os desvios padrões entre os resultados da decisão (Tabela 2) gerados por cada um foi pequeno. Isso revela um ponto importante, de que a escolha do método não deve influenciar significativamente no resultado da decisão.

\section{Agradecimentos}

Ao programa CNPq/PIBIC pelo financiamento do projeto de pesquisa, à UNICAMP pela concessão da bolsa de Iniciação Científica e ao meu orientador, Prof. Dr. Leonardo Tomazeli Duarte, por todo suporte fornecido.

1 A.T. de Almeida; D.C. Morais; A.P.C.S. Costa; L.H. Alencar; and S.F.D. Daher. Decisão em grupo e negociação: métodos e aplicações. Atlas, São Paulo, 2012.

2 L. Yu, K.K. Lai. A distance-based group decision-making methodology for multi-person multi-criteria emergency decision support. Decision Support Systems, 51 (2011) 307-315.

3 Salvatore Greco. Multiple criteria decision analysis: state of the art surveys, volume 78. Springer, 2004. 